Mathematical Research Letters 3, 211-229 (1996)

\title{
K3 SURFACES, LORENTZIAN KAC-MOODY ALGEBRAS AND MIRROR SYMMETRY
}

\author{
Valeri A. Gritsenko and Viacheslav V. Nikulin
}

\begin{abstract}
A bitract. We consider the variant of the Mirror Symmetry Conjecture for K3 surfaces which relates "geometry" of curves on a general member of a family of K3 surfaces with "algebraic functions" on the moduli of the mirror family. Lorentzian Kac-Moody algebras are involved in this construction. We give several examples when this conjecture is valid.
\end{abstract}

\section{Introduction}

In this paper we want to interpret our results [GN1], [GN2] and [N10] from the viewpoint of mirror symmetry for K3 surfaces.

This paper was written during our stay at SFB 170 "Geometrie und Analysis" of Mathematical Institute of Georg-August-University in September - October 1995. We are grateful to the Mathematical Institute for hospitality.

\section{Mirror symmetry for K3 surfaces}

Let $S$ be an even hyperbolic lattice, i.e., a free $\mathbb{Z}$-module of rank $n+1$ with an integral even symmetric bilinear form of signature $(1, n)$. This lattice $S$ may appear in two ways in connection with algebraic K3 surfaces:

(A) $S=S_{X}$ is the Picard lattice of a K3 surface $X$. These K3 surfaces form a family

$$
\mathcal{M}_{S}=\left\{\text { K3 surface } X \mid S \subset S_{X}\right\}
$$

of dimension $20-\operatorname{dim} S$. See [N1], [N6] for definition which is actually based on local (G.N. Tjurina in [ $\breve{\mathrm{S}}]$ ) and global Torelli Theorem [P-S $\breve{S}-\breve{S}]$ and epimorphicity of Torelli map $[\mathrm{Ku}]$ for K3 surfaces. A general member $X$ of this family has the Picard lattice $S_{X}=S$.

Received October 12, 1995.

The first author was supported by SFB 170 "Geometrie und Analysis".

This second author was partially supported by a grant of the Russian Fund of Fundamental Research; Grant of ISF MI6000; Grant of ISF and Russian Government MI6300; Grant of AMS; SFB 170 "Geometrie und Analysis". 
(B) $S=\left([c]_{T}^{\perp}\right) /[c]$, for the lattice of transcendental cycles $T=T_{X}$ (the transcendental lattice) of a K3 surface $X$ where $c \in T$ is a primitive element of $T$ with $c^{2}=0$. (We consider K3 surfaces over $\mathbb{C}$; then $T_{X}=$ $\left(S_{X}\right)_{H^{2}(X, \mathbb{Z})}^{\perp}$.) These K3 surfaces $X$ form a family

$$
\mathcal{M}_{T^{\perp}}=\left\{\text { K3 surface } X \mid T_{X} \subset T\right\}
$$

of dimension $\operatorname{dim} S$. A general member $X$ of this family has $T_{X}=T$.

These two families $\mathcal{M}_{S}$ and $\mathcal{M}_{T \perp}$ are called dual (or mirror symmetric, or mirror). This is how mirror symmetry for K3 surfaces (inspired by explanation of the Arnold's strange duality [A] for 14 exceptional unimodal singularities of functions) had first appeared in $[\mathrm{P}],[\mathrm{DN}]$ and $[\mathrm{N} 2],[\mathrm{D} 1]$. In particular, in [N2] there was developed some lattice theory for the exact calculation of these dual families. The new understanding of mirror symmetry for K3 (see for example [D2]) which is due to the modern physics and mirror symmetry for Calabi-Yau threefolds (see [COGP] and [Mor1], [Mor2]) is related with the fact that one can calculate the lattice $S$ for the situation (B) using Yukawa coupling at the point defined by $c$ at infinity of $\mathcal{M}_{T^{\perp}}$. Moreover, there are no quantum corrections for the model (A).

For the model (A), the lattice $S$ is related with the geometry of curves and is the intersection form of all curves on a general K3 surface $X \in$ $\mathcal{M}_{S}$. For the model (B), the lattice $S$ is related with the geometry of moduli $\mathcal{M}_{T^{\perp}}$ at an appropriate point at infinity of the mirror (dual) family $\mathcal{M}_{T^{\perp}} \subset \overline{\mathcal{M}}_{T^{\perp}}$. Thus, for any question related with the geometry of curves on a general member $X$ of the family $\mathcal{M}_{S}$, one can ask about its analog for the dual family $\mathcal{M}_{T^{\perp}}$ from the point of view of the geometry of the moduli $\mathcal{M}_{T^{\perp}}$.

An effect we want to discuss here is the following:

It turns out that in some cases "geometry" of irreducible and effective classes of divisors on general $X \in \mathcal{M}_{S}$ is related with interesting "algebraic functions" on the dual family $\mathcal{M}_{T^{\perp}}$. This relation involves Lorentzian KacMoody Lie algebras (and conjecturally some physics).

Here an element of $S=S_{X}$ is called irreducible (respectively effective) if it contains an irreducible (respectively effective) curve. The moduli $\mathcal{M}_{T^{\perp}}$ is a quotient of a Hermitian symmetric domain of type IV by some arithmetic group $G$, and "algebraic function" means here an automorphic form with respect to $G$ on this domain.

\section{Geometry of irreducible and effective classes of divisors on a K3 surface}

In this section, we consider a hyperbolic lattice $S$ from the point of view of the model (A). Thus, now $S=S_{X}$ is the Picard lattice of a K3 surface 
$X$. Then elements of $S$ reflect some geometry of curves. An element $h \in S$ is called irreducible if it contains an irreducible curve on $X$. An element $h \in S$ is called effective if it is a finite sum of irreducible elements. For K3 surfaces, the sets of the effective and irreducible classes may be described purely arithmetically using only the intersection form of the lattice $S$ (up to automorphisms of $S$ ). Now we give this description.

It is sufficient to describe the set $\Delta^{\text {ir }} \subset S$ of all irreducible elements. It is well-known (and very easy to see) that $h^{2} \geq-2$ if $h \in \Delta^{\text {ir }}$. In particular, $\Delta^{\mathrm{ir}}=\Delta_{-2}^{\mathrm{ir}} \cup \Delta_{\geq 0}^{\mathrm{ir}}$ where $\Delta_{-2}^{\mathrm{ir}}=\left\{\delta \in \Delta^{\mathrm{ir}} \mid \delta^{2}=-2\right\}, \Delta_{\geq 0}^{\mathrm{ir}}=\left\{h \in \Delta^{\mathrm{ir}} \mid h^{2} \geq\right.$ $0\}$. An element $\delta$ belongs to $\Delta_{-2}^{\mathrm{ir}}$ if and only if it contains a non-singular irreducible rational curve (exceptional curve) on $X$. An element $h \in S$ is called nef if $h \cdot C \geq 0$ for any irreducible curve $C$ on $X$. We denote by $\operatorname{NEF}(S)$ the set of all nef elements of $S$. It is known that the sets $\operatorname{NEF}(S)$ and $\Delta_{\geq 0}^{\mathrm{ir}}$ almost coincide. Obviously, $x^{2} \geq 0$ if $x \in \operatorname{NEF}(S)$, and $\mathbb{N} \Delta_{\geq 0}^{\mathrm{ir}} \subset \operatorname{NEF}(X)$. If $c \in \operatorname{NEF}(S)$ and $c^{2}=0$, then $c \in \Delta_{\geq 0}^{\mathrm{ir}}$ if and only if $c$ is primitive [P- $\breve{S}-\breve{S}$ ]. If $h \in \operatorname{NEF}(S)$ and $h^{2}>0$, then $h \in \Delta_{\geq 0}^{\text {ir }}$ if and only if there does not exist primitive $c \in \operatorname{NEF}(S)$ with $c^{2}=0$ such that $c \cdot h=1$ (in particular, $2 h \in \Delta_{>0}^{\mathrm{ir}}$ ). See [SD]. Thus, the set $\Delta_{\geq 0}^{\mathrm{ir}}$ is completely determined by the set $\operatorname{NEF}(S)$. In what follows, we use the set $\operatorname{NEF}(S)$ instead of $\Delta_{>0}^{\mathrm{ir}}$ since it is more convenient to work with.

Since the lattice $S$ is hyperbolic, the cone

$$
V(S \otimes \mathbb{R})=\left\{x \in S \otimes \mathbb{R} \mid x^{2}>0\right\}
$$

is the union of two half cones $\pm V^{+}(S \otimes \mathbb{R})$ where $V^{+}(S \otimes \mathbb{R})$ contains the class of a hyperplane section. It is easy to see that

$$
\operatorname{NEF}(S)=\left\{h \in S \mid h \in \overline{V^{+}(S \otimes \mathbb{R})}-\{0\}, h \cdot \Delta_{-2}^{\mathrm{ir}} \geq 0\right\} .
$$

Thus, $\operatorname{NEF}(S)$ is completely defined by $\Delta_{-2}^{\mathrm{ir}}$. Moreover, there exists a group-theoretical description of both sets. Let

$$
\mathbb{R}_{++} \mathcal{M}=\left\{x \in \overline{V^{+}(S \otimes \mathbb{R})}-\{0\} \mid x \cdot \Delta_{-2}^{\text {ir }} \geq 0\right\}
$$

be a cone and $\mathcal{M}=\mathbb{R}_{++} \mathcal{M} / \mathbb{R}_{++}$its set of rays. Then $\operatorname{NEF}(S)=S \cap$ $\mathbb{R}_{++} \mathcal{M}$. Let $W^{(2)}(S) \subset O(S)$ be the group generated by all reflections $s_{\delta}: x \mapsto x+(x \cdot \delta) \delta, x \in S$, of the lattice $S$ in elements $\delta \in S$ with $\delta^{2}=-2$. It is easy to see that the group $W^{(2)}(S)$ is discrete in the corresponding hyperbolic space $\mathcal{L}^{+}(S)=V^{+}(S) / \mathbb{R}_{++}$and $\mathcal{M}$ is the fundamental domain of $W^{(2)}(S)$ with the set $\Delta_{-2}^{\text {ir }}$ of vectors orthogonal to $\mathcal{M}$. It means that $\delta \in \Delta_{-2}^{\text {ir }}$ if and only if $\delta \in S, \delta^{2}=-2$ and the inequality $\delta \cdot x \geq 0$ defines 
a face of $\mathcal{M}$ (or of $\mathbb{R}_{++} \mathcal{M}$ ) of codimension one. This gives the description of the both sets $\operatorname{NEF}(S)$ and $\Delta_{-2}^{\text {ir }}$ of $S$ in terms of the group $W^{(2)}(S)$ : the real convex cone $\mathbb{R}_{++} \operatorname{NEF}(S)$ is a fundamental domain for the group $W^{(2)}(S)$ acting on $V^{+}(S \otimes \mathbb{R})$ with the set of orthogonal vectors $\Delta_{-2}^{\text {ir }}$.

Let $\operatorname{EF}(S)$ be the set of all effective elements of $S$ and $\operatorname{EF}(S)_{\geq-2}$, $\operatorname{EF}(S)_{-2}$ and $\operatorname{EF}(S)_{\geq 0}$ are the sets of all elements $x \in \operatorname{EF}(S)$ with $x^{2} \geq-2$, $x^{2}=-2$ and $x^{2} \geq 0$ respectively. Using Riemann-Roch Theorem, one can see that

$$
\begin{aligned}
& \operatorname{EF}(S)_{-2}=\left\{\delta \in S \mid \delta^{2}=-2, \delta \cdot \operatorname{NEF}(S) \geq 0\right\}, \\
& \operatorname{EF}(S)_{\geq 0}=S \cap \overline{V^{+}(S \otimes \mathbb{R})}-\{0\}, \quad \text { and, } \\
& \operatorname{EF}(S)_{\geq-2}=\operatorname{EF}(S)_{-2} \cup \operatorname{EF}(S)_{\geq 0} .
\end{aligned}
$$

\section{Kac-Moody algebras associated to a K3 surface}

In this section, we define Kac-Moody algebras associated to a K3 surface $X$ with the Picard lattice $S=S_{X}$. (See [Ka1], [Ka2], [Ka3], [Bo1] and [GN1] for the theory of Kac-Moody algebras.) Such an algebra will be a generalized Kac-Moody (Lie) superalgebra without odd real simple roots. It is defined by a set ${ }_{s} \Delta$ of simple roots which is divided in a set of simple real (even) $\operatorname{roots}{ }_{s} \Delta^{\text {re }}$ and a set of simple imaginary roots ${ }_{s} \Delta^{\mathrm{im}}$; the last set is divided in a set of even simple imaginary roots ${ }_{s} \Delta_{\overline{0}}^{\mathrm{im}}$ and a set of simple odd imaginary roots ${ }_{s} \Delta_{\overline{1}}^{\mathrm{im}}$.

We put ${ }_{s} \Delta^{\mathrm{re}}=\Delta_{-2}^{\mathrm{ir}}$ and the sets ${ }_{s} \Delta_{\overline{0}}^{\mathrm{im}},{ }_{s} \Delta_{\overline{1}}^{\mathrm{im}}$ are some sequences of nef elements of $S$. Each imaginary root $\alpha$ defines an element of $\operatorname{NEF}(S)$ but one can repeat each element of $\operatorname{NEF}(S)$ finite number of times in each set ${ }_{s} \Delta_{\overline{0}}^{\mathrm{im}}$ and ${ }_{s} \Delta_{\overline{1}}^{\mathrm{im}}$.

The generalized Kac-Moody superalgebra without odd real simple roots $\mathfrak{g}=\mathfrak{g}^{\prime \prime}\left(S,{ }_{s} \Delta^{\mathrm{im}}\right)$ is a Lie superalgebra generated by $h_{r}, e_{r}, f_{r}$ where $r \in$ ${ }_{s} \Delta^{\mathrm{im}}$. All $h_{r}$ are even, $e_{r}, f_{r}$ are even (respectively odd) if $r$ is even (respectively odd). The algebra $\mathfrak{g}$ has the following defining relations:

(1) The map $r \mapsto h_{r}$ for $r \in{ }_{s} \Delta$ gives an embedding of $S \otimes \mathbb{R}$ into $\mathfrak{g}^{\prime \prime}\left(S,{ }_{s} \Delta^{\mathrm{im}}\right)$ as an abelian subalgebra (it is even since all $h_{r}$ are even). In particular, all elements $h_{r}$ commute.

(2) $\left[h_{r}, e_{r^{\prime}}\right]=-\left(r \cdot r^{\prime}\right) e_{r^{\prime}}$, and $\left[h_{r}, f_{r^{\prime}}\right]=\left(r \cdot r^{\prime}\right) f_{r^{\prime}}$.

(3) $\left[e_{r}, f_{r^{\prime}}\right]=h_{r}$ if $r=r^{\prime}$, and is 0 if $r \neq r^{\prime}$.

(4) $\left(\operatorname{ad} e_{r}\right)^{1+r \cdot r^{\prime}} e_{r^{\prime}}=\left(\operatorname{ad} f_{r}\right)^{1+r \cdot r^{\prime}} f_{r^{\prime}}=0$ if $r \neq r^{\prime}$ and $r \in{ }_{s} \Delta^{\mathrm{re}}$.

(5) If $r \cdot r^{\prime}=0$, then $\left[e_{r}, e_{r^{\prime}}\right]=\left[f_{r}, f_{r^{\prime}}\right]=0$.

The superalgebra $\mathfrak{g}=\mathfrak{g}^{\prime \prime}\left(S,{ }_{s} \Delta^{\mathrm{im}}\right)$ is graded by $S$ as follows. Let

$$
\widetilde{Q}_{+}=\sum_{\alpha \in \in_{s} \Delta} \mathbb{Z}_{+} \alpha \subset S
$$


be the integral cone (semi-group) generated by all simple roots. We have

$$
\mathfrak{g}=\left(\bigoplus_{\alpha \in Q_{+}-\{0\}} \mathfrak{g}_{\alpha}\right) \bigoplus \mathfrak{g}_{0} \bigoplus\left(\bigoplus_{\alpha \in Q_{+}-\{0\}} \mathfrak{g}_{-\alpha}\right)
$$

where $h_{r}$ has degree 0 , and $e_{r}$ and $f_{r}$ have degree $r \in \widetilde{Q}_{+}$and $-r \in-\widetilde{Q}_{+}$ respectively $\left(r \in{ }_{s} \Delta\right)$; and $\mathfrak{g}_{0}=S \otimes \mathbb{R}$. A non-zero $\alpha \in \pm \widetilde{Q}_{+}$is called a root if $\mathfrak{g}_{\alpha}$ is non-zero. Let $\Delta$ be the set of all roots and $\Delta_{ \pm}=\Delta \cap \pm \widetilde{Q}_{+}$. For a root $\alpha \in \Delta$ we set mult $\overline{0} \alpha=\operatorname{dim} \mathfrak{g}_{\alpha, \overline{0}}, \operatorname{mult}_{\overline{1}} \alpha=-\operatorname{dim}_{\mathfrak{g}_{\alpha, \overline{1}}}$ and

$$
\text { mult } \alpha=\operatorname{mult}_{\overline{0}} \alpha+\operatorname{mult}_{\overline{1}} \alpha=\operatorname{dim} \mathfrak{g}_{\alpha, \overline{0}}-\operatorname{dim} \mathfrak{g}_{\alpha, \overline{1}} \text {. }
$$

The integer mult $\alpha$ is called the multiplicity of $\alpha$. According to the general theory of Kac-Moody algebras, the set of roots is the union of real and imaginary roots: $\Delta=\Delta^{\mathrm{re}} \cup \Delta^{\mathrm{im}}$. The set of real roots is $\Delta^{\mathrm{re}}=W^{(2)}(S)\left({ }_{s} \Delta^{\mathrm{re}}\right)$ (in particular, $\alpha^{2}=-2$ if $\alpha \in \Delta^{\mathrm{re}}$ ). The set of imaginary roots is $\Delta^{\mathrm{im}}=\left\{\alpha \in \Delta \mid \alpha^{2} \geq 0\right\}$. It follows that $\Delta_{+}^{\text {re }}:=$ $\Delta^{\mathrm{re}} \cap \Delta_{+}=\operatorname{EF}(S)_{-2}$ and $\Delta_{+}^{\mathrm{im}}:=\Delta^{\mathrm{im}} \cap \Delta_{+} \subset \operatorname{EF}(S)_{\geq 0}$. If $\alpha \in \Delta^{\mathrm{re}}$, then mult $_{\overline{0}} \alpha=1$, mult $\overline{\overline{1}}_{\overline{1}} \alpha=0$ and mult $\alpha=1$. Thus, we can rewrite the decomposition above using "geometry" of K3 surfaces as follows:

$$
\mathfrak{g}=\left(\bigoplus_{\alpha \in \operatorname{EF}(S)_{\geq-2}} \mathfrak{g}_{\alpha}\right) \bigoplus \mathfrak{g}_{0} \bigoplus\left(\bigoplus_{\alpha \in \operatorname{EF}(S)_{\geq-2}} \mathfrak{g}_{-\alpha}\right)
$$

Here $\mathfrak{g}_{\alpha}=0$ if $\alpha \notin \Delta$.

Since the lattice $S$ is hyperbolic, the algebra $\mathfrak{g}=\mathfrak{g}^{\prime \prime}\left(S,{ }_{s} \Delta^{\mathrm{im}}\right)$ is Lorentzian. Moreover ${ }_{s} \Delta^{\text {re }}$ is the whole set $\Delta_{-2}^{\text {ir }}$, therefore the algebra $\mathfrak{g}$ has restricted arithmetic type (see [N10]). Thus, the algebras associated to a K3 surface are Lorentzian generalized Kac-Moody superalgebras of restricted arithmetic type without odd real simple roots.

In what follows, we restrict ourselves to considering $S$ with a lattice Weyl vector for $\Delta_{-2}^{\mathrm{ir}}$.

Definition. An element $\rho \in S \otimes \mathbb{Q}$ is called a lattice Weyl vector if $\rho \cdot \delta=1$ for any $\delta \in{ }_{s} \Delta^{\mathrm{re}}=\Delta_{-2}^{\mathrm{ir}}$.

There are three cases when a lattice Weyl vector does exist:

(i) $\Delta_{-2}^{\text {ir }}=\emptyset$, then we can take any $\rho \in S \otimes \mathbb{Q}$;

(ii) $\operatorname{dim} S=2$ and $\Delta_{-2}^{\text {ir }} \neq \emptyset$, then the set $\Delta_{-2}^{\text {ir }}$ is linearly independent and does not contain more than 2 elements; 
(iii) $\operatorname{dim} S \geq 3, \Delta_{-2}^{\mathrm{ir}} \neq \emptyset$ and a lattice Weyl vector $\rho$ exists. It follows from general results [N4], [N5] and [N10], that the set of hyperbolic lattices $S$ with this property is finite up to isomorphism. These lattices $S$ are divided in two classes. Firstly, it is easy to see that $\rho$ is a nef element of $S$ and for large $n \in \mathbb{N}$ the linear system $|h|$ of $h=n \rho \in S$ is free. If $\rho^{2}>0$ (this case is called elliptic), the linear system $|h|$ gives an embedding of $X$ into a projective space such that all non-singular rational curves on $X$ have the same degree $n$. All these cases are known (see [N3], [N7], [N8]). If $\rho^{2}=0$ (this case is called parabolic), then $|h|$ gives an elliptic fibration of $X$ over a projective line such that all non-singular rational curves of $X$ have the same degree $n$ over the projective line. The list of such $S$ is not known yet.

We would like to mention that in the case (iii) the fundamental polyhedron $\mathcal{M}=\mathbb{R}_{++} \operatorname{NEF}(S) / \mathbb{R}_{++}$for the action of $W^{(2)}(S)$ on the hyperbolic space $\mathcal{L}^{+}(S)$ is a very right and beautiful polyhedron: it is a fundamental polyhedron for a reflection group and it touches a sphere with centre $\mathbb{R}_{++} \rho$.

The case (iii) is especially interesting for us because it is very exceptional: there is only finite number of possibilities. Moreover, we want to get some relations between the sets $\operatorname{NEF}(S)$ and $\operatorname{EF}(S)$ which are different only in the cases (ii) and (iii). The case (iii) is also related with automorphic forms on multi-dimensional (dim $\geq 3$ ) Hermitian domains.

From now on we assume that $S$ has a lattice Weyl vector $\rho$. For $a \in$ $\operatorname{NEF}(S)$, let $m(a)_{\overline{0}}^{\prime}, m(a)_{\overline{1}}^{\prime}$ are equal to the numbers of times we repeat $a$ in the sequences ${ }_{s} \Delta_{\overline{0}}^{\mathrm{im}}$ and ${ }_{s} \Delta_{\overline{1}}^{\mathrm{im}}$ respectively. We set $m(a)^{\prime}=m(a)_{\overline{0}}^{\prime}-m(a)_{\overline{1}}^{\prime}$. Let $a_{0}$ be a primitive element of $\operatorname{NEF}(S)$ with $a_{0}^{2}=0$. In this case we define "corrected" invariants $m\left(t a_{0}\right), t \in \mathbb{N}$, using the identity of power series:

$$
\prod_{n \in \mathbb{N}}\left(1-q^{n}\right)^{m\left(n a_{0}\right)^{\prime}}=1-\sum_{t \in \mathbb{N}} m\left(t a_{0}\right) q^{t}
$$

For $a \in \operatorname{NEF}(S)$ with $a^{2}>0$ we set $m(a)=m(a)^{\prime}$.

We have the following Weyl-Kac-Borcherds denominator identity for Kac-Moody superalgebra $\mathfrak{g}=\mathfrak{g}^{\prime \prime}\left(S,{ }_{s} \Delta^{\mathrm{im}}\right)$ (see [Ka1], [Bo1], and [GN1]):

$$
\begin{aligned}
& \Phi(z) \\
& =\sum_{w \in W^{(2)}(S)} \operatorname{det}(w)\left(\exp (2 \pi i(w(\rho) \cdot z))-\sum_{a \in \operatorname{NEF}(S)} m(a) \exp (2 \pi i(w(\rho+a) \cdot z))\right) \\
& =\exp (2 \pi i(\rho \cdot z)) \prod_{\alpha \in \operatorname{EF}(S)_{\geq-2}}(1-\exp (2 \pi i(\alpha \cdot z)))^{\text {mult } \alpha},
\end{aligned}
$$


where $z$ belongs to the complexified cone $\Omega(S)=S \otimes \mathbb{R}+i V^{+}(S \otimes \mathbb{R})$ of $V^{+}(S \otimes \mathbb{R})$. The function $\Phi(z)$ is called the denominator function of $\mathfrak{g}=\mathfrak{g}^{\prime \prime}\left(S,{ }_{s} \Delta^{\mathrm{im}}\right)$.

Considering different sequences ${ }_{s} \Delta^{\mathrm{im}}$ of simple imaginary roots from $\operatorname{NEF}(S)$ we get different denominator identities which one can consider as multi-dimensional identities relating the sets of effective and nef (or irreducible) elements of $S$. Actually these identities depend only on the integral function $m(a), a \in \operatorname{NEF}(S)$. If this function is given, one can calculate $m(a)^{\prime}$ and find all possible non-negative integers $m(a)_{\frac{1}{0}}^{\prime}, m(a)_{\frac{1}{1}}^{\prime}$ with $m(a)^{\prime}=m(a)_{\frac{1}{0}}^{\prime}-m(a)_{\frac{1}{1}}^{\prime}$ which define Kac-Moody superalgebras $\mathfrak{g}=$ $\mathfrak{g}^{\prime \prime}\left(S,{ }_{s} \Delta^{\mathrm{im}}\right)$ with the fixed denominator function. One also can consider the function $\left(^{*}\right)$ as some kind of integral

$$
\Phi(z)=\int_{C \subset X} \xi(C, z)
$$

along effective curves on the K3 surface $X$ with $S_{X}=S$. This integral could be correctly defined because we only use effective classes in the formula $(*)$.

\section{A variant of mirror conjecture}

Now we consider the hyperbolic lattice $S$ using the model (B). We consider only the simplest case when

$$
T=S \oplus U(k), \quad k \in \mathbb{N}, \quad U(k)=\left(\begin{array}{cc}
0 & k \\
k & 0
\end{array}\right)
$$

Let $c_{1}, c_{2}$ be a basis of $U(k)$ with this intersection matrix. Then $z \mapsto$ $\mathbb{C}\left(z \oplus\left(-z^{2} / 2\right) c_{1} \oplus(1 / k) c_{2}\right)$ defines an embedding corresponding to the cusp defined by $c_{1}$ of the complexified cone $\Omega(S)$ to the connected component $\Omega(T)_{0}$ of the Hermitian symmetric domain of type IV

$$
\Omega(T)=\left\{\mathbb{C} \omega \subset T \otimes \mathbb{C} \mid \omega^{2}=0, \omega \cdot \bar{\omega}>0\right\}
$$

The choice of $\omega_{0}=z \oplus\left(-z^{2} / 2\right) c_{1} \oplus(1 / k) c_{2} \in \mathbb{C} \omega_{0}$ is determined by the normalization $\omega_{0} \cdot c_{1}=1$. For this normalization, the local moduli of K3 are identified with $S \otimes \mathbb{C}$ and Yukawa coupling coincides with the intersection pairing of the lattice $S$. This normalization is prescribed by mirror symmetry for K3. The quotient $\left(\mathcal{M}_{T^{\perp}}\right)_{0}=O(T)^{\prime} \backslash \Omega(T)_{0}$ is a connected component of the dual (mirror) family of K3 surfaces for an appropriate subgroup $O(T)^{\prime}$ of finite index of $O(T)$. 
Mirror Conjecture. There exists a choice of $k \in \mathbb{N}$ and a sequence ${ }_{s} \Delta^{\mathrm{im}} \subset N E F(S)$ of simple imaginary roots such that the denominator function $\Phi(z)$ of $\mathfrak{g}^{\prime \prime}\left(S,{ }_{s} \Delta^{\mathrm{im}}\right)$ is a holomorphic automorphic form with respect to $O(T)^{\prime}$ on the domain $\Omega(T)_{0}$, i.e. $\Phi(z)$ is an "algebraic function" on the dual moduli $\mathcal{M}_{T^{\perp}}$ (model $\left.(B)\right)$. The form $\Phi(z)$ has the following sense from the point of view of the model $(A): \Phi(z)$ is written in the form $(*)$ using "geometry of curves" (effective and irreducible or nef classes of divisors) of a general member $X$ with $S_{X}=S$ of the family $\mathcal{M}_{S}$ and it gives an identity $(*)$ between effective and nef divisor classes on $X$. Moreover, we suppose that for the automorphic form $\Phi(z)$ it is possible to give exact formulae for Fourier coefficients $m(a)$ of the left side and multiplicities mult $\alpha$ of the right side of $\left(^{*}\right)$. Besides, the generalized Kac-Moody superalgebra $\mathfrak{g}^{\prime \prime}\left(S,{ }_{s} \Delta^{\mathrm{im}}\right)$ should also be related with geometry of curves and moduli of K3 (and conjecturally with some physics).

It is very important that the zero divisor of $\Phi(z)$ in the domain where the product $\left(^{*}\right)$ converges has multiplicity one and is contained in the discriminant

$$
\mathcal{D}=O(T)^{\prime} \backslash\left(\underset{\delta \in T, \delta^{2}=-2}{\bigcup} D_{\delta}\right)
$$

of moduli $\mathcal{M}_{T^{\perp}}$ of K3 surfaces where $D_{\delta}=\{\mathbb{C} \omega \in \Omega(T) \mid \omega \cdot \delta=0\}$. Therefore, in some sense, $\Phi(z)$ shows how far we are from the discriminant.

In the rest part of the paper we give several examples when this conjecture is valid.

\section{Example 1}

For the first example, $\operatorname{dim} S=3, S \cong U(4) \oplus\langle-2\rangle$. (In what follows we denote by $K(t)$ a lattice which one gets by multiplication on $t \in \mathbb{Q}$ of the symmetric bilinear form of the lattice $K$.) The set $\Delta_{-2}^{\mathrm{ir}}(S)=\left\{\delta_{1}, \delta_{2}, \delta_{3}\right\}$ generates the lattice $S$ and has the intersection matrix

$$
\left(\delta_{i} \cdot \delta_{j}\right)=\left(\begin{array}{rrr}
-2 & 2 & 2 \\
2 & -2 & 2 \\
2 & 2 & -2
\end{array}\right)
$$

which defines the lattice $S$. The fundamental polyhedron $\mathcal{M}$ is the right triangle with the vertices at infinity. The lattice Weyl vector $\rho$ is equal to $\rho=\left(\delta_{1}+\delta_{2}+\delta_{3}\right) / 2$. The element $h=2 \rho$ has the square $h^{2}=6$ and the linear system $|h|$ gives an embedding of a K3 surface $X$ with $S_{X}=S$ as an intersection of a quadric and a cubic in $\mathbb{P}^{4}$. For this embedding, all non-singular rational curves on $X$ are three conics corresponding to 
$\delta_{1}, \delta_{2}, \delta_{3}$. Their sum is a hyperplane section of $X$. The lattice $T$ is equal to $T=U(4) \oplus S \cong 2 U(4) \oplus\langle-2\rangle$. The orthogonal complement $T^{\perp}$ is isomorphic to a hyperbolic lattice $S^{\prime} \cong U(4) \oplus K$ where $K$ is a negative definite lattice of rank 15 with the discriminant quadratic form $q_{U(4)} \oplus q_{\langle 2\rangle}$. It follows from results of [N2] that the lattice $S^{\prime}$ is unique and the moduli space of K3 surfaces $\mathcal{M}_{S^{\prime}}$ is irreducible. (It would be very interesting to determine this family using equations and to give an algebraic description of the automorphic form $F_{1}(Z)$ which we shall describe. We hope to do this later.)

Let us consider another basis $f_{2}, f_{3}, f_{-2}$ of $S \otimes \mathbb{Q}$ where

$$
\delta_{1}=2 f_{2}-f_{3}, \delta_{2}=2 f_{-2}-f_{3}, \quad \delta_{3}=f_{3} .
$$

These elements have the intersection matrix

$$
\left(f_{i} \cdot f_{j}\right)=\left(\begin{array}{rrr}
0 & 0 & 1 \\
0 & -2 & 0 \\
1 & 0 & 0
\end{array}\right) .
$$

Thus the lattice $S$ is a sublattice of $M_{0}=\mathbb{Z} f_{2} \oplus \mathbb{Z} f_{3} \oplus \mathbb{Z} f_{-2}$ of index 4 . We have $M_{0}=U \oplus\langle-2\rangle$ where $U=\mathbb{Z} f_{2} \oplus \mathbb{Z} f_{-2}$ and $\mathbb{Z} f_{3}=\langle-2\rangle$. These lattices are related as follows: $S \cong 2\left(M_{0}\right)^{*}$. We consider coordinates $\left(z_{3}, z_{2}, z_{1}\right)$ where $z=z_{3} f_{2}+z_{2} f_{3}+z_{1} f_{-2} \in M_{0} \otimes \mathbb{C}=S \otimes \mathbb{C}$. We introduce the lattice $L=U \oplus M_{0}$ where $U=\mathbb{Z} f_{1} \oplus \mathbb{Z} f_{-1}$ with $f_{1}^{2}=f_{-1}^{2}=0$ and $f_{1} \cdot f_{-1}=1$. We use $z$ as a coordinate for the point $Z=\mathbb{C}\left(\left(-z^{2} / 2\right) f_{1}+z+f_{-1}\right) \in \Omega(L)$ of the domain $\Omega(L)$ of the type IV corresponding to $L$. We also identify $Z$ with the matrix

$$
Z=\left(\begin{array}{ll}
z_{1} & z_{2} \\
z_{2} & z_{3}
\end{array}\right) \in \mathbb{H}_{2}
$$

where $\mathbb{H}_{2}$ is the Siegel upper-half plane.

Let us consider the classical function $\Delta_{5}(Z)$ (see $[\mathrm{F}]$ ) which is the product of all even theta-constants

$$
\Delta_{5}(Z)=\prod_{(a, b)} \vartheta_{a, b}(Z), \quad\left(Z=\left(\begin{array}{cc}
z_{1} & z_{2} \\
z_{2} & z_{3}
\end{array}\right) \in \mathbb{H}_{2}\right)
$$

with

$$
\vartheta_{a, b}(Z)=\sum_{l \in \mathbb{Z}^{2}} \exp \left(\pi i\left(Z\left[l+\frac{1}{2} a\right]+{ }^{t} b l\right)\right) \quad\left(Z[l]={ }^{t} l Z l\right) .
$$

The product is taken over all vectors $a, b \in(\mathbb{Z} / 2 \mathbb{Z})^{2}$ such that ${ }^{t} a b \equiv$ $0 \bmod 2$. (There are exactly ten different $(a, b)$.) This is the automorphic cusp form of weight 5 with a character with respect to $S p_{4}(\mathbb{Z}) /\left\{ \pm E_{4}\right\} \cong$ $O^{+}(L) /\left\{ \pm E_{5}\right\}$ where $O^{+}(L)$ is the subgroup of $O(L)$ which fixes two connected components of $\Omega(L)$ (see [GN1]). The function $F_{1}(Z)=\frac{1}{64} \Delta_{5}(Z)$ has integral Fourier coefficients. 
Theorem 1. The function $F_{1}(Z)$ gives the solution of Mirror Conjecture of $\S 4$ for the lattice $S$ and $U(4)=\mathbb{Z} c_{1} \oplus \mathbb{Z} c_{2}$ where $c_{1}=2 f_{1}, c_{2}=2 f_{-1}$. Therefore $F_{1}(Z)$ is an "algebraic function" on the moduli $\mathcal{M}_{T^{\perp}}$ where $T=$ $U(4) \oplus S$, and it defines the identity $\left(^{*}\right)$ for $S=S_{X}$ of the general member $X$ of the mirror family $\mathcal{M}_{S}$. Moreover, $F_{1}(Z)$ defines the corresponding Kac-Moody superalgebras $\mathfrak{g}^{\prime \prime}\left(S,{ }_{s} \Delta^{\mathrm{im}}\right)$.

Proof. The function $F_{1}(Z)$ as a function on $\Omega(L)$ is automorphic with respect to $O^{+}(L)$. We have the equality $T=2 L^{*}$ because $U(4)=2 U^{*}$ and $S=2\left(M_{0}\right)^{*}$. It follows that $F_{1}(Z)$ is automorphic with respect to $O^{+}(T)=O^{+}(L)$ and defines then an "algebraic function" on the moduli $\mathcal{M}_{T^{\perp}}=O^{+}(T) \backslash \Omega(T)$.

It is proved in [GN1] that for the coordinate $z$ which we introduced above, the function $F_{1}(Z)$ can be written in the form

$$
\begin{aligned}
& F_{1}(Z) \\
& =\sum_{w \in W^{(2)}(S)} \operatorname{det}(w)\left(\exp (\pi i(w(\rho) \cdot z))-\sum_{a \in \operatorname{NEF}(S)} m(a) \exp (\pi i(w(\rho+a) \cdot z))\right) \\
& =\exp (\pi i(\rho \cdot z)) \prod_{\alpha \in \operatorname{EF}(S)_{\geq-2}}(1-\exp (\pi i(\alpha \cdot z)))^{\text {mult } \alpha}
\end{aligned}
$$

with integral coefficients $m(a)$ and mult $\alpha$. For $c_{1}=2 f_{1}$ and $k=4$, we should consider the coordinate $z^{\prime}=z / 2$ (mirror symmetry coordinate) instead of the coordinate $z$. For this coordinate $z^{\prime}$, from (5.1) we get

$$
\begin{aligned}
& F_{1}(Z) \\
& =\sum_{w \in W^{(2)}(S)} \operatorname{det}(w)\left(\exp \left(2 \pi i\left(w(\rho) \cdot z^{\prime}\right)\right)-\sum_{a \in \operatorname{NEF}(S)} m(a) \exp \left(2 \pi i\left(w(\rho+a) \cdot z^{\prime}\right)\right)\right) \\
& =\exp \left(2 \pi i\left(\rho \cdot z^{\prime}\right)\right) \prod_{\alpha \in \operatorname{EF}(S)_{\geq-2}}\left(1-\exp \left(2 \pi i\left(\alpha \cdot z^{\prime}\right)\right)\right)^{\text {mult } \alpha} .
\end{aligned}
$$

This proves Theorem 1.

To get an exact formula for the coefficients $m(a)$ and mult $\alpha$ in (5.1), we need two types of Jacobi forms. The Jacobi form of the first type is the form of weight 5 and index $1 / 2$

$$
\psi_{5, \frac{1}{2}}\left(z_{1}, z_{2}\right)=\eta\left(z_{1}\right)^{9} \vartheta_{11}\left(z_{1}, z_{2}\right) .
$$


Here $\eta\left(z_{1}\right)=q^{1 / 24} \prod_{n \geq 1}\left(1-q^{n}\right)$ is Dedekind eta-function and

$$
\begin{aligned}
\vartheta_{11}\left(z_{1}, z_{2}\right) & =\sum_{n \in \mathbb{Z}}(-1)^{n} \exp \left(\frac{\pi i}{4}(2 n+1)^{2} z_{1}+\pi i(2 n+1) z_{2}\right) \\
& =-q^{1 / 8} r^{-1 / 2} \prod_{n \geq 1}\left(1-q^{n-1} r\right)\left(1-q^{n} r^{-1}\right)\left(1-q^{n}\right)
\end{aligned}
$$

is the classical Jacobi theta-function, where we put

$$
\begin{gathered}
z_{1} \in \mathbb{H}_{1}=\left\{z_{1}=x+i y \in \mathbb{C} \mid y>0\right\}, z_{2} \in \mathbb{C} \\
q=\exp \left(2 \pi i z_{1}\right), r=\exp \left(2 \pi i z_{2}\right), p=\exp \left(2 \pi i z_{3}\right) .
\end{gathered}
$$

The holomorphic function $\psi_{5, \frac{1}{2}}\left(z_{1}, z_{2}\right)$ is a Jacobi form of index one-half with a multiplier system. It means that the following identities are satisfied

$$
\begin{gathered}
\psi_{5, \frac{1}{2}}\left(\frac{a z_{1}+b}{c z_{1}+d}, \frac{z_{2}}{c z_{1}+d}\right)=v_{\eta}^{12}(g)\left(c z_{1}+d\right)^{5} \exp \left(\pi i \frac{c z_{2}^{2}}{c z_{1}+d}\right) \psi_{5, \frac{1}{2}}\left(z_{1}, z_{2}\right) \\
\psi_{5, \frac{1}{2}}\left(z_{1}, z_{2}+p z_{1}+q\right)=(-1)^{p+q} \exp \left(-\pi i\left(p^{2} z_{1}+2 p z_{2}\right)\right) \psi_{5, \frac{1}{2}}\left(z_{1}, z_{2}\right)
\end{gathered}
$$

where $p, q \in \mathbb{Z}, g=\left(\begin{array}{ll}a & b \\ c & d\end{array}\right) \in S L_{2}(\mathbb{Z})$ and

$$
\eta\left(\frac{a z_{1}+b}{c z_{1}+d}\right)=v_{\eta}(g)\left(c z_{1}+d\right)^{1 / 2} \eta\left(z_{1}\right) .
$$

Here $v_{\eta}(g)$ is a 24 th root of unity.

Let us consider the Fourier coefficients of $\eta^{d}\left(z_{1}\right)$

$$
q^{d / 24} \prod_{n \geq 1}\left(1-q^{n}\right)^{d}=\sum_{m} \tau_{d}(m) q^{m / 24}
$$

Then we have the following Fourier expansion of $\psi_{5, \frac{1}{2}}\left(z_{1}, z_{2}\right)$ :

$$
\begin{aligned}
\psi_{5, \frac{1}{2}}\left(z_{1}, z_{2}\right) & =\eta\left(z_{1}\right)^{9} \vartheta_{11}\left(z_{1}, z_{2}\right) \\
& =\sum_{\substack{n, l \equiv 1 \bmod 2 \\
n>0,4 n-l^{2}>0}}(-1)^{\frac{l-1}{2}} \tau_{9}\left(4 n-l^{2}\right) \exp \left(\pi i\left(n z_{1}+l z_{2}\right)\right) .
\end{aligned}
$$

The second type of Jacobi forms which we need are special Jacobi forms of weight zero (weak Jacobi forms in terms of [EZ]). The ring of all weak 
Jacobi forms has two generators as an algebra over $S L_{2}(\mathbb{Z})$-modular forms (see $[\mathrm{EZ}, \S 9]$ ). One of these generators is the function

$$
\phi_{0,1}\left(z_{1}, z_{2}\right)=\frac{1}{144 \Delta\left(z_{1}\right)}\left(E_{4}^{2}\left(z_{1}\right) E_{4,1}\left(z_{1}, z_{2}\right)-E_{6}\left(z_{1}\right) E_{6,1}\left(z_{1}, z_{2}\right)\right)
$$

where

$$
\begin{aligned}
\Delta\left(z_{1}\right) & =q \prod_{n}\left(1-q^{n}\right)^{24}, \\
E_{4}\left(z_{1}\right) & =1+240 \sum_{n \geq 1} \sigma_{3}(n) q^{n}, \\
E_{6}\left(z_{1}\right) & =1-504 \sum_{n \geq 1} \sigma_{5}(n) q^{n},
\end{aligned}
$$

are the cusp forms of weight 12 and the Eisenstein series of weights 4 and 6 for $S L_{2}(\mathbb{Z})$. $E_{k, 1}\left(z_{1}, z_{2}\right)$ is the Jacobi-Eisenstein series of weight $k$ and index one which has the following integral Fourier coefficients (see [EZ, §2])

$$
E_{k, 1}\left(z_{1}, z_{2}\right)=\zeta(3-2 k)^{-1} \sum_{\substack{n, l \in \mathbb{Z} \\ 4 n-l^{2} \geq 0}} H\left(k-1,4 n-l^{2}\right) q^{n} r^{l},
$$

where $H(k, N)$ are H. Cohen's numbers (see [C]). If $D=(-1)^{k} N$ is a discriminant of a quadratic field, then $H(k, N)=L(1-k,(\underline{D}))$ are values of the Dirichlet $L$-function with the character $\chi_{D}(n)=\left(\frac{D}{n}\right)$. We recall that $\zeta(-5)=-1 / 252$ and $\zeta(-9)=-1 / 132$.

The weak Jacobi form $\phi_{0,1}$ has the Fourier expansion with integral coefficients

$$
\begin{aligned}
\phi_{0,1}\left(z_{1}, z_{2}\right) & =\sum_{\substack{n, l \in \mathbb{Z}, n \geq 0 \\
4 n-l^{2} \geq-1}} f(n, l) \exp \left(2 \pi i\left(n z_{1}+l z_{2}\right)\right) \\
& =\left(r^{-1}+10+r\right)+q\left(10 r^{-2}-64 r^{-1}+108-64 r+10 r^{2}\right)+\ldots
\end{aligned}
$$

which depend only on the "norm" $4 n-l^{2}$ of $(n, l)$

$$
f(n, l)=c_{1}\left(4 n-l^{2}\right) \quad \text { and } \quad c_{1}(m)=0 \text { for } m<-1 .
$$

Moreover, the function

$$
C_{1}\left(z_{1}\right)=\sum_{\substack{m \geq-1 \\ m \equiv 0,3 \bmod 4}} c_{1}(m) q^{m}
$$


is an automorphic form of weight $-\frac{1}{2}$. It is easy to get a formula for this function using the Cohen's modular forms of half integral weight $k-\frac{1}{2}$

$$
\mathcal{H}_{k-1}\left(z_{1}\right)=\sum_{n \geq 0} H(k-1, n) q^{n}
$$

(see $[\mathrm{C}]$ and $[\mathrm{EZ}, \S 5])$. One has

$$
\begin{aligned}
\Delta\left(4 z_{1}\right) C_{1}\left(z_{1}\right) & =\frac{1}{12}\left(11 E_{6}\left(4 z_{1}\right) \mathcal{H}_{5}\left(z_{1}\right)-21 E_{8}\left(4 z_{1}\right) \mathcal{H}_{3}\left(z_{1}\right)\right) \\
& =q^{3}+10 q^{4}+\cdots
\end{aligned}
$$

We would like to note that the function:

$$
11 E_{6}\left(4 z_{1}\right) \mathcal{H}_{5}\left(z_{1}\right)-21 E_{8}\left(4 z_{1}\right) \mathcal{H}_{3}\left(z_{1}\right)
$$

is a cusp form of weight $11 \frac{1}{2}$ for $\Gamma_{0}(4)$.

Using the functions introduced above and Theorem 4.1 of [GN1], we can write the identity (5.1) in the following form:

$$
\begin{aligned}
& F_{1}(Z) \\
& =\sum_{\substack{n, l, m \equiv 1 \bmod 2 \\
n, m>0}} \sum_{d \mid(n, l, m)}(-1)^{\frac{l+d+2}{2}} \tau_{9}\left(\frac{4 n m-l^{2}}{d^{2}}\right) q^{n / 2} r^{l / 2} p^{m / 2} \\
& =(q r p)^{1 / 2} \prod_{\substack{n, l, m \in \mathbb{Z} \\
(n, l, m)>0}}\left(1-q^{n} r^{l} p^{m}\right)^{c_{1}\left(4 n m-l^{2}\right)},
\end{aligned}
$$

where $(n, l, m)>0$ means that $n \geq 0, m \geq 0$, and $l$ is an arbitrary integral if $n+m>0$, and $l<0$ if $n=m=0$.

\section{Example 2}

For this example, $\operatorname{dim} S=3$ and $S \cong U(8) \oplus\langle-2\rangle$. The set $\Delta_{-2}^{\mathrm{ir}}(S)=$ $\left\{e_{1}, e_{2}, e_{3}, e_{4}\right\}$ generates the lattice $S$ and has the intersection matrix

$$
\left(e_{i} \cdot e_{j}\right)=\left(\begin{array}{rrrr}
-2 & 2 & 6 & 2 \\
2 & -2 & 2 & 6 \\
6 & 2 & -2 & 2 \\
2 & 6 & 2 & -2
\end{array}\right)
$$


which defines the lattice $S$. The fundamental polyhedron $\mathcal{M}$ is the right quadrangle with the vertices at infinity. The lattice Weyl vector $\rho$ is given by the equality $\rho=\left(e_{1}+e_{3}\right) / 4=\left(e_{2}+e_{4}\right) / 4$. The element $h=4 \rho$ has the square $h^{2}=8$ and the linear system $|h|$ gives an embedding of a K3 surface $X$ with $S_{X}=S$ as an intersection of three quadrics in $\mathbb{P}^{5}$ (this follows easily from general results of $[\mathrm{SD}]$ ). For this embedding, all four non-singular rational curves on $X$ have degree 4 . The curves $e_{1}+e_{3}$ and $e_{2}+e_{4}$ give two hyperplane sections of $X$. The lattice $T$ is $T=U(8) \oplus S \cong$ $2 U(8) \oplus\langle-2\rangle$. The orthogonal complement $T^{\perp}$ is isomorphic to a hyperbolic lattice $S^{\prime} \cong U(8) \oplus K$ where $K$ is a negative definite lattice of the rank 15 with the discriminant quadratic form $q_{U(8)} \oplus q_{\langle 2\rangle}$. It follows from results of [N2], that the lattice $S^{\prime}$ is unique and the moduli space $\mathcal{M}_{S^{\prime}}$ is irreducible.

We describe below an automorphic form connected to the lattice $S$. This form gives the solution of the mirror conjecture in $\S 4$ in this case. We consider a hyperbolic lattice $M_{0}$ with the basis $f_{2}, f_{3}, f_{-2}$ having the intersection matrix

$$
\left(f_{i} \cdot f_{j}\right)=\left(\begin{array}{rrr}
0 & 0 & 1 \\
0 & -4 & 0 \\
1 & 0 & 0
\end{array}\right) .
$$

Let us take the hyperbolic plane $U$ with the standard basis $f_{1}, f_{-1}$ where $f_{1}^{2}=f_{-1}^{2}=0, f_{1} \cdot f_{-1}=1$, the lattice $L=U \oplus M_{0}$ and the domain $\Omega(L)$. We use the coordinate $z^{\prime}=z_{3}^{\prime} f_{2}+z_{2}^{\prime} f_{3}+z_{1}^{\prime} f_{-2}$ for a point $Z^{\prime}=$ $\mathbb{C}\left(\left(-\left(z^{\prime}\right)^{2} / 2\right) f_{1}+z^{\prime}+f_{-1}\right)$ in this domain. We define

$$
\left\{\delta_{1}=-f_{3}, \delta_{2}=4 f_{2}+f_{3}, \delta_{3}=4 f_{2}+3 f_{3}+4 f_{-2}, \delta_{4}=f_{3}+4 f_{-2}\right\} \subset M_{0} .
$$

Then $\delta_{i} \cdot \delta_{j}=2 e_{i} \cdot e_{j}$. Thus, the sublattice $M_{I I} \subset M_{0}$ generated by $\delta_{1}, \ldots, \delta_{4}$ is isomorphic to $S(2) \cong U(16) \oplus\langle-4\rangle$. Equivalently, $S=M_{I I}(1 / 2)$. We identify this lattices replacing $e_{i}$ by $\delta_{i}$.

In [GN1, §5] there was constructed an automorphic cusp form $F_{2}\left(Z^{\prime}\right)$ of weight 2 with a character with respect to $O^{+}(L) /\left\{ \pm E_{5}\right\}$. This function has the following representation with integral coefficients

$$
\begin{aligned}
& F_{2}\left(Z^{\prime}\right) \\
& =\sum_{w \in W^{(2)}(S)} \operatorname{det}(w)\left(\exp \left(\frac{\pi i}{2}\left(w(\rho) \cdot z^{\prime}\right)\right)-\sum_{a \in \operatorname{NEF}(S)} m(a) \exp \left(\frac{\pi i}{2}\left(w(\rho+a) \cdot z^{\prime}\right)\right)\right) \\
& =\exp \left(\frac{\pi i}{2}\left(\rho \cdot z^{\prime}\right)\right) \prod_{\alpha \in \operatorname{EF}(S)_{\geq-2}}\left(1-\exp \left(\frac{\pi i}{2}\left(\alpha \cdot z^{\prime}\right)\right)\right)^{\text {mult } \alpha} .
\end{aligned}
$$


Theorem 2. The function $F_{2}\left(Z^{\prime}\right)$ gives the solution of Mirror Conjecture of $\S 4$ for the lattice $S=M_{I I}(1 / 2) \cong 2 U(8) \oplus\langle-2\rangle$ and $U(8)=\left[\mathbb{Z} c_{1} \oplus\right.$ $\left.\mathbb{Z} c_{2}\right](1 / 2)$ where $c_{1}=4 f_{1}, c_{2}=4 f_{-1}$. Therefore, $F_{2}(Z)$ is an "algebraic function" on the moduli $\mathcal{M}_{T^{\perp}}($ model $(B))$ where $T=U(8) \oplus S$, and it defines the identity $\left(^{*}\right)$ for $S=S_{X}$ of the general member $X$ of the mirror family $\mathcal{M}_{S}$ (model $\left.(A)\right)$. Moreover, it defines the corresponding Kac-Moody superalgebras $\mathfrak{g}^{\prime \prime}\left(S,{ }_{s} \Delta^{\mathrm{im}}\right)$.

Proof. The function $F_{2}\left(Z^{\prime}\right)$ as a function on $\Omega(L)$ is automorphic with respect to $O^{+}(L)$. We have $T(2)=4 L^{*}$ because $U(16)=4 U^{*}$ and $M_{I I}=$ $4\left(M_{0}\right)^{*}$. It follows that $F_{2}\left(Z^{\prime}\right)$ is automorphic with respect to $O^{+}(T)=$ $O^{+}(T(2))=O^{+}(L)$ and defines an "algebraic function" on the moduli $\mathcal{M}_{T^{\perp}}=O^{+}(T) \backslash \Omega(T)$.

For $c_{1}=4 f_{1}, c_{2}=4 f_{-1}$ and $U(8)$ we should use the mirror symmetry coordinate $z^{\prime \prime}=z^{\prime} / 2$. We should also remember that $S=M_{I I}(1 / 2)$. From (6.1), we get the identity

$$
\begin{aligned}
& F_{2}\left(Z^{\prime}\right) \\
& =\sum_{w \in W^{(2)}(S)} \operatorname{det}(w)\left(\exp \left(2 \pi i\left(w(\rho) \cdot z^{\prime \prime}\right)\right)-\sum_{a \in \operatorname{NEF}(S)} m(a) \exp \left(2 \pi i\left(w(\rho+a) \cdot z^{\prime \prime}\right)\right)\right) \\
& =\exp \left(2 \pi i\left(\rho \cdot z^{\prime \prime}\right)\right) \prod_{\alpha \in \operatorname{EF}(S)_{\geq-2}}\left(1-\exp \left(2 \pi i\left(\alpha \cdot z^{\prime \prime}\right)\right)\right)^{\text {mult } \alpha},
\end{aligned}
$$

where we use the intersection pairing of the lattice $S$ and $z^{\prime \prime} \in S \otimes \mathbb{C}$. This proves Theorem 2 .

The function $F_{2}\left(Z^{\prime}\right)$ (see $[\mathrm{GN}, \S 5]$ ) is connected with the Jacobi functions

$$
\begin{aligned}
\phi_{0,2}\left(z_{1}, z_{2}\right) & =\frac{1}{288 \Delta_{12}\left(z_{1}\right)}\left(E_{4}\left(z_{1}\right) E_{4,1}^{2}\left(z_{1}, z_{2}\right)-E_{6,1}^{2}\left(z_{1}, z_{2}\right)\right) \\
& =\sum_{n, l} c_{2}\left(8 n-l^{2}\right) \exp \left(2 \pi i\left(n z_{1}+l z_{2}\right)\right)
\end{aligned}
$$

and

$$
\begin{aligned}
\psi_{2, \frac{1}{2}}\left(z_{1}, z_{2}\right) & =-\eta^{3}(\tau) \vartheta_{11}\left(z_{1}, z_{2}\right) \\
& \left.=\sum_{n \equiv 1 \bmod 4 l \equiv 1 \bmod 2}(-1)^{\frac{l+1}{2}} \tau_{3}\left(2 n-l^{2}\right) \exp \left(\frac{\pi i}{2} n z_{1}+\pi i l z_{2}\right)\right) .
\end{aligned}
$$


The coefficients $\tau_{3}(n)$ are given by the Jacobi formula

$$
\eta^{3}\left(z_{1}\right)=\sum_{m \geq 1}\left(\frac{-4}{m}\right) m q^{m^{2} / 8}
$$

where

$$
\left(\frac{-4}{m}\right)=\left\{\begin{aligned}
1 & \text { if } m \equiv 1 \bmod 4 \\
-1 & \text { if } m \equiv-1 \bmod 4 \\
0 & \text { if } m \equiv 0 \bmod 2
\end{aligned}\right.
$$

is the generalized symbol of the quadratic residue. The numbers $c_{2}(n)$, which define the Fourier coefficients of the Jacobi form $\psi_{2, \frac{1}{2}}$, are Fourier coefficients of an automorphic form of weight $-1 / 2$. One can express them in terms of Cohen's numbers $H(3, N)$ and $H(5, N)$. Using these functions we can rewrite the identity (6.1) in the following form (see [GN1, §5])

$$
\begin{aligned}
& F_{2}\left(Z^{\prime}\right) \\
& =\sum_{N \geq 1} \sum_{\substack{2 m n-l^{2}=N^{2} \\
n, m \equiv 1 \bmod 4 \\
n>0, l \equiv 1 \bmod 2,}}(-1)^{\frac{l+1}{2}}\left(\frac{-4}{N}\right) N \sum_{d \mid(n, l, m)}\left(\frac{-4}{d}\right) q^{n / 4} r^{l / 2} p^{m / 4} \\
& =q^{1 / 4} r^{-1 / 2} p^{1 / 4} \prod_{\substack{n, l, m \in \mathbb{Z} \\
(n, l, m)>0}}\left(1-q^{n} r^{l} p^{m}\right)^{c_{2}\left(8 n m-l^{2}\right)},
\end{aligned}
$$

where $(n, l, m)>0$ means that $n \geq 0, m \geq 0, l$ is an arbitrary integral if $n+m>0$, and $l>0$ if $n=m=0 ; \quad q=\exp \left(2 \pi i z_{1}^{\prime}\right), r=\exp \left(2 \pi i z_{2}^{\prime}\right)$, $p=\exp \left(2 \pi i z_{3}^{\prime}\right)$.

Let us consider the coordinate $Z=\left(\begin{array}{ll}z_{1} & z_{2} \\ z_{2} & z_{3}\end{array}\right) \in \mathbb{H}_{2}$ where $z_{1}=z_{1}^{\prime}$, $z_{2}=z_{2}^{\prime}, z_{3}=z_{3}^{\prime} / 2$. The function $F_{2}(Z)$ is the cusp form of weight 2 with a character (with values in the group of fourth roots of unity) for the double extension of the paramodular group

$$
\Gamma_{2}:=\left\{\left(\begin{array}{cccc}
* & 2 * & * & * \\
* & * & * & 2^{-1} * \\
* & 2 * & * & * \\
2 * & 2 * & 2 * & *
\end{array}\right) \in S p_{4}(\mathbb{Q}), \quad \text { all } * \in \mathbb{Z}\right\} .
$$

This function is a lifting of Jacobi form $\psi_{2, \frac{1}{2}}$ (see [G2], [G3], [G4]). 


\section{Example 3}

For this example, $\operatorname{dim} S=10$ and $S \cong U \oplus E_{8}(2)$. Let $U=\mathbb{Z} c \oplus \mathbb{Z} e$ where $c^{2}=0, e^{2}=-2$ and $c \cdot e=1$. Then

$$
\Delta_{-2}^{\mathrm{ir}}=\left\{\delta \in S \mid \delta^{2}=-2, \delta \cdot c=1\right\} .
$$

For example, $e \in \Delta_{-2}^{\text {ir }}$. This case is parabolic and $\rho=c$. For a K3 surface $X$ with $S_{X}=S$, we have $|\rho|: X \rightarrow \mathbb{P}^{1}$ is elliptic fibration. All non-singular rational curves on $X$ are sections of this fibration. Probably, this family of K3 surfaces had first appeared in [N3] (see also [N8]) where $X \in \mathcal{M}_{S}$ were described as follows. There exists an involution $\sigma$ on $X$ such that $H^{2}(X, \mathbb{Z})^{\sigma}=S$. This involution is unique on $X$ and $\sigma^{*} \omega_{X}=-\omega_{X}$. The set of points of $X$ fixed by this involution is union of two non-singular fibers (two elliptic curves) of the fibration $|\rho|$ above. Let $Y$ be a K3 surface with involution $\sigma$ on $Y$ such that the set of points of $Y$ fixed by this involution is union of two elliptic curves. Then $S \cong H^{2}(Y, \mathbb{Z})^{\sigma} \subset S_{Y}$ and $Y$ belongs to $\mathcal{M}_{S}$.

We can interpret the results of [Bo3] as a construction of a function $F_{3}(Z)$ which gives the solution of Mirror Conjecture in $\S 4$ for $U(2)(k=$ $2)$. Thus, for this case, $T=U(2) \oplus S \cong U(2) \oplus U(1) \oplus E_{8}(2)$. Then $S^{\prime}=T^{\perp} \cong U(2) \oplus E_{8}(2)$ and $\mathcal{M}_{S^{\prime}}$ is the family of K3 surfaces which are universal coverings of Enriques surfaces ("Enriques family"). In other words, $X \in \mathcal{M}_{S^{\prime}}$ has an involution $\tau$ without fixed points. Then $X /\{1, \tau\}$ is an Enriques surface.

\section{Questions}

It would be interesting to formulate the Mirror Conjecture of $\S 4$ for hyperbolic lattices $S$ which do not have a lattice Weyl vector for $\Delta_{-2}^{\text {ir }}$. It is certainly possible for some cases. Is this possible for arbitrary hyperbolic lattices $S$ ? What is an analog of the Mirror Conjecture in $\S 4$ for Calabi-Yau threefolds?

\section{References}

[A] V. I. Arnold, Critical points of smooth functions and their normal forms, Uspehi Mat. Nauk 30 (1975), no. 5, 3-65; English transl. in Russian Math. Surveys 30 (1975).

[Bo1] R. Borcherds, Generalized Kac-Moody algebras, J. of Algebra 115 (1988), 501512 .

[Bo2] Automorphic forms on $O_{s+2,2}(R)$ and infinite products, Invent. Math. 120 (1995), 161-213.

[Bo3] - The moduli space of Enriques surfaces and the fake monster Lie superalgebra, preprint (1994). 
[C] H. Cohen, Sums involving the values at negative integers of L-functions of quadratic characters, Math. Ann. 217 (1975), 271-285.

[COGP] P. Candelas, X. C. de la Ossa, P. S. Green, and L. Parkes, A Pair of CalabiYau manifolds as an exactly soluble Ssuperconformal theory, Nucl. Phys. B359 (1991), 21-74.

[D1] I. V. Dolgachev, Integral quadratic forms: applications to algebraic geometry (after V. Nikulin), Sem. Bourbaki, 1982/83, n. 611, Astérisque 105/106, 251275.

[D2] _ Mirror symmetry for lattice polarized K3-surfaces, Duke e-prints alggeom 9502005 (1995).

[DN] I. V. Dolgachev and V. V. Nikulin, The exceptional singularities of V.I. Arnold and K3 surfaces, Seventh All-Union Topology Conf. (Minsk, 1977), Abstracts of Lectures and Communications, Inst. Mat. Akad. Nauk BSSR, Minsk (Russian) (1977).

[EZ] M. Eichler and D. Zagier, The theory of Jacobi forms, Progress in Math. 55, Birkhäuser, 1985.

[G1] V. A. Gritsenko, Jacobi functions in n-variables, Zap. Nauk. Sem. LOMI 168 (1988), 32-45 (Russian); English transl. in J. Soviet Math. 53 (1991), 243-252.

[G2] - Modular forms and moduli spaces of abelian and K3 surfaces, Mathematica Gottingensis Schrift. des SFB "Geometrie und Analysis", Heft 26, 1993, pp. 32; St.Petersburg Math. Jour. 6:6 (1994), 65-102.

[G3] - Irrationality of the moduli spaces of polarized abelian surfaces, The International Mathematics Research Notices 6 (1994), 235-243, In full form in "Abelian varieties", Proc. of the Egloffstein conference, de Gruyter, Berlin, 1995, pp. 63-81.

[G4] Modulformen zur Paramodulgruppe und Modulräume der Abelschen Varietäten, Mathematica Gottingensis Schrift. des SFB "Geometrie und Analysis", Heft 12 (1995), 1-89.

[GN1] V. A. Gritsenko and V. V. Nikulin, Siegel automorphic form correction of some Lorentzian Kac-Moody Lie algebras, Duke e-prints alg-geom 9504006 (1995).

[GN2] Siegel automorphic form correction of a Lorentzian Kac-Moody algebra, C. R. Acad. Sci. Paris Sér. A-B 321 (1995), 1151-1156.

[F] E. Freitag, Siegelsche Modulfunktionen, Springer, 1983.

[Ka1] V. Kac, Infinite dimensional Lie algebras, Cambridge Univ. Press, 1990.

[Ka2] L Lie superalgebras, Adv. Math. 26 (1977), 8-96.

[Ka3] _ Infinite-dimensional algebras, Dedekind's $\eta$-function, classical Möbius function and the very strange formula, Adv. Math. 30 (1978), 85-136.

$[\mathrm{Ku}] \quad$ Vic. S. Kulikov, Degenerations of K3 surfaces and Enriques surfaces, Izv. Akad. Nauk SSSR Ser. Mat. 41 (1977), 1008-1042; English transl. in Math. USSR Izv. 11 (1977).

[Mor1] D. R. Morrison, Mirror symmetry and rational curves on quintic threefolds: $A$ guide for mathematicians, J. Amer. Math. Soc. 6 (1993), 223-247.

[Mor2] C Compactifications of moduli spaces inspired by mirror symmetry, Journées de Géométrie Algébrique d'Orsay (Juillet 1992), Astérisque 218 (1993), 243271.

[N1] V. V. Nikulin, Finite automorphism groups of Kähler K3 surfaces, Trudy Moskov. Mat. Obshch. 37 (1979), 73-137; English transl. in Trans. Moscow Math. Soc. 38 (1980), no. 2. 
[N2] Integral symmetric bilinear forms and some of their applications, Izv. Akad. Nauk SSSR Ser. Mat. 43 (1979), 111-177; English transl. in Math. USSR Izv. 14 (1980).

[N3] On the quotient groups of the automorphism groups of hyperbolic forms by the subgroups generated by 2-reflections, Algebraic-geometric applications, Current Problems in Math. Vsesoyuz. Inst. Nauchn. i Tekhn. Informatsii, Moscow (1981), 3-114; English transl. in J. Soviet Math. 22 (1983), 1401-1476.

[N4] - On arithmetic groups generated by reflections in Lobachevsky spaces, Izv. Akad. Nauk SSSR Ser. Mat. 44 (1980), 637-669; English transl. in Math. USSR Izv. 16 (1981).

[N5] - On the classification of arithmetic groups generated by reflections in Lobachevsky spaces, Izv. Akad. Nauk SSSR Ser. Mat. 45 (1981), no. 1, 113-142; English transl. in Math. USSR Izv. 18 (1982).

[N6] Involutions of integral quadratic forms and their applications to real algebraic geometry, Izv. Akad. Nauk SSSR Ser. Mat. 47 (1983), no. 1; English transl. in Math. USSR Izv. 22 (1984), 99-172.

[N7] _ Surfaces of type K3 with finite automorphism group and Picard group of rank three, Proc. Steklov. Math. Inst. 165 (1984), 113-142; English transl. in Trudy Inst. Steklov 3 (1985).

[N8] _ Discrete reflection groups in Lobachevsky spaces and algebraic surfaces, Proc. Int. Congr. Math. Berkeley 1986, vol. 1, pp. 654-669.

[N9] - A lecture on Kac-Moody Lie algebras of the arithmetic type, Preprint Queen's University, Canada \#1994-16, (1994), or Duke e-prints alg-geom 9412003.

[N10] , Reflection groups in hyperbolic spaces and the denominator formula for Lorentzian Kac-Moody Lie algebras, Duke e-prints alg-geom 9503003 (1995) (to appear in Russian Acad. Sci. Izv. Math.).

[P] H. C. Pinkham, Singularités exceptionnelles, la dualité étrange d'Arnold et les surfaces K-3, C. R. Acad. Sci. Paris Sér. A-B 284 (1977), A615-A618.

[P- $\breve{S}-\breve{S}]$ I. I. Pjatetckii-S̆apiro and I. R. S̆afarevich, A Torelli theorem for algebraic surfaces of type K3, Izv. Akad. Nauk SSSR Ser. Mat. 35 (1971), 530-572; English transl. in Math. USSR Izv. 5 (1971).

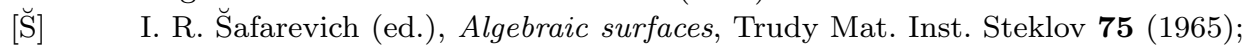
English transl. in Proc. Steklov Inst. Math. 75 (1965).

[SD] B. Saint-Donat, Projective models of K-3 surfaces, Amer. J. Math. 96 (1974), 602-639.

St. Petersiurg Department Steklov M athematical institute Fontanka 27,191011 ST. PEtersburg, Russia

E-mail address: gritsenk@gauss.pdmi.ras.ru, valera@mathi.uni-heidelberg.de

Steklov Mathematical Institute, ul. Vavilova 42, M oscow 117966 , GSP-1, R USSIA.

E-mail address: slava@nikulin.mian.su 